\title{
Antithrombotic therapy in heart failure
}

\author{
F. W. A. Verheugt
}

Published online: 10 February 2012

(C) Springer Media / Bohn Stafleu van Loghum 2012

\begin{abstract}
Patients with congestive heart failure have a significant risk of stroke due to thromboembolism from the dilated left ventricle. Two relatively small trials suggest that oral anticoagulation with vitamin-K antagonists may reduce this risk when compared with placebo, aspirin or clopidogrel. However, more studies are eagerly awaited. So far, physicians seeing patients with heart failure should decide who needs antithrombotic prophylaxis on a case-by-case basis, especially since most heart failure patients have significant comorbidity precluding the use of oral anticoagulant.
\end{abstract}

Keywords Heart failure · Stroke · Thromboembolism . Warfarin $\cdot$ Aspirin $\cdot$ Clopidogrel

\section{Pathophysiology of thromboembolism in heart failure}

Patients with congestive heart failure have a significant risk for malignant arrhythmia, and for thromboembolic complications. Due to the diminished blood flow in the dilated atria and ventricles, these chambers are thought to be responsible for the development of thrombi, which can subsequently be embolised. The risk of thromboembolism is further enhanced by the presence of atrial fibrillation, which is common in patients with heart failure. But also patients with left ventricular dysfunction and sinus rhythm are at significant risk of thromboembolic complications and might benefit

F. W. A. Verheugt $(\bowtie)$

Department of Cardiology, Onze Lieve Vrouwe Gasthuis (OLVG), Oosterpark 9,

1091 AC, Amsterdam, the Netherlands

e-mail: f.w.a.verheugt@olvg.nl from oral anticoagulation, as observed in a large patient cohort in the SOLVD trial [1]. Formerly, oral anticoagulation was a common secondary prevention after myocardial infarction. Although some physicians prescribe warfarin after anterior myocardial infarction complicated by left ventricular aneurysm (which is not evidence-based), oral anticoagulation after myocardial infarction is nowadays largely replaced by aspirin and clopidogrel. Many heart failure patients have underlying ischaemic heart disease and are, therefore, treated with aspirin.

One disadvantage of aspirin is its theoretical interaction with angiotensin-converting enzyme (ACE) inhibitors, a standard therapy in heart failure. Aspirin is thought to block kidney prostaglandin production leading to elevated blood pressure. Previous observations have addressed this and found a decreased long-term mortality benefit of ACE inhibitors for in 6 placebo-controlled trials in patients with or without aspirin [2] (Table 1). However, until the WATCH and WASH trials [3-5] there had not been a randomised study investigating aspirin in heart failure patients on ACE inhibitors.

Randomised trials of antithrombotic therapy in heart failure

The American WATCH trial evaluated the optimal antithrombotic strategy for thromboembolism prevention in patients with heart failure in sinus rhythm and the clinical interactions between aspirin and ACE inhibitors [4]. The trial was planned to include 4500 patients, but randomisation was prematurely discontinued due to slow recruitment. In total only 1587 heart failure patients with sinus rhythm were randomised to $162 \mathrm{mg}$ aspirin daily, to $75 \mathrm{mg}$ clopidogrel daily or to warfarin with a target international normalised ratio between 2.5 and 3.0 (median achieved 2.6). After 23 months of follow-up, there were no differences in the occurrences of the primary endpoint myocardial 
Table 1 Long-term risk of death on aspirin and no aspirin in six randomised post-myocardial infarction trials with ACE inhibitors [2]

* for interaction aspirin versus no aspirin; : $O R$ odds ratio; $C I$ confidence interval

\begin{tabular}{lcccc}
\hline & ACE inhibitor & Control & OR (99\% CI) & p \\
\hline Patients $(n=22,060)$ & $17.7 \%$ & $20.7 \%$ & $0.82(0.75-0.89)$ & \\
Aspirin $(n=14,410)$ & $16.2 \%$ & $18.1 \%$ & $0.86(0.77-0.97)$ & \\
No aspirin $(n=7650)$ & $20.4 \%$ & $25.5 \%$ & $74(0.64-0.86)$ & $0.04^{*}$ \\
\hline
\end{tabular}

infarction, stroke or death. Stroke occurred in $0.7 \%$ of the warfarin patients, compared with $2.1 \%$ of aspirin and $2.5 \%$ of clopidogrel patients $(p<0.05$ versus aspirin/clopidogrel combined). Hospitalisation for heart failure was seen in $16.1 \%$ of the warfarin group, $22.2 \%$ of the aspirin group $(p<0.01$ versus warfarin) and $18.3 \%$ of the clopidogrel group ( $p=0.38$ versus warfarin). Cerebral bleeding was most common on warfarin therapy $(1.3 \%)$, but this was not significantly different from aspirin or clopidogrel $(0.6 \%$ and $0.5 \%$, respectively). As expected, also severe extracranial bleeding was most frequent in the warfarin patients $(5.6 \%)$ and was seen less with clopidogrel and aspirin $(2.5 \%$ and $3.1 \%$, respectively). The WATCH trial did not address whether antithrombotic therapy is necessary in heart failure in the first place, since it had no placebo group.

The WASH trial carried out in the United Kingdom studied the role of warfarin, aspirin or no prophylaxis in 279 ACE inhibitor-treated patients with heart failure in sinus rhythm to prevent stroke, myocardial infarction and death [5]. Although the sample size was too small to be conclusive, there was no difference in the primary endpoints between groups. However, hospitalisation for heart failure was seen in $58 \%$ of aspirin patients versus $42 \%$ on warfarin and $48 \%$ without prophylaxis $(p=0.05)$. Stroke occurred in no warfarin patients, compared with $2 \%$ on aspirin and $2 \%$ without prophylaxis. Expectedly, severe bleeding was seen in $4 \%$ on warfarin, $1 \%$ on aspirin and none without prophylaxis. Despite the numbers in WASH being small, warfarin was the best therapy for preventing stroke and hospitalisation for heart failure, but at the cost of increased bleeding complications.

Neither WATCH nor WASH answer the question of what the optimal antithrombotic prophylaxis should be, if needed. All patients were in sinus rhythm, but most had coronary artery disease. A placebo-controlled comparison was not, therefore, deemed ethically acceptable in the large WATCH study, although it was used WASH.

Both studies show that for patients receiving ACE inhibitors for heart failure the inhibition of prostaglandin production by aspirin may lead to more hospital admissions than for patients not taking aspirin. By contrast, clopidogrel does not seem to have this disadvantage in WATCH.
These are important findings, although in many European countries the daily aspirin dose is under $160 \mathrm{mg}$ and, therefore, the unfavourable results of aspirin in both trials (162 mg in WATCH and $300 \mathrm{mg}$ in WASH) may be of limited value. Furthermore, in a large American registry of 24,012 elderly patients with heart failure with coronary artery disease the benefit of aspirin on 1-year mortality (Table 2) and readmission for heart failure was not influenced by the concomitant use of ACE of inhibitors [6].

The above registry does of course lack the power of randomised trials, but unfortunately the WATCH and WASH trials are insufficiently powered to support warfarin or clopidogrel and discourage aspirin use and, thus, change current practice in heart failure and sinus rhythm. The observational data $[2,6]$ suggest that aspirin-associated (re)admissions for heart failure, if any, do not translate into increased risk for death. It is most unlikely that a trial with clopidogrel alone in the prevention of stroke and thromboembolism in heart failure will ever be done.

Better answers may come from the WARCEF (Warfarin versus Aspirin in Reduced Ejection Fraction) study, which included 3200 patients with a left ventricular ejection fraction $<35 \%$ and sinus rhythm and may be reported later this year. Stroke and death will be the primary endpoint, and myocardial infarction and hospitalisation for heart failure are prespecified secondary endpoints.

Table 2 One-year risk of death on aspirin $(n=13,049)$ and no aspirin $(n=$ 10,963 ) in elderly patients taking ACE inhibitors for heart failure [6]

Adjusted RR (95\%

CI)

\begin{tabular}{ll}
\hline $\begin{array}{l}\text { All patients }(n=24,012) \\
\text { Patients on ACE inhibitors }(53 \%)\end{array}$ & $0.94(0.89-0.99)$ \\
& $0.94(0.87-1.01)$ \\
$\begin{array}{l}\text { Patients without ACE inhibitors } \\
(47 \%)\end{array}$ & $0.93(0.87-1.00)$
\end{tabular}

* $p$ for interaction for ACE inhibitors or no ACE inhibitors; $R R$ relative risk; $C I$ confidence intervals; $n s$ not significant 


\section{Conclusion}

Given the above data physicians seeing patients with heart failure should decide who needs antithrombotic prophylaxis on a case-by-case basis, especially since many heart failure patients do have significant comorbidity precluding the use of oral anticoagulants.

\section{References}

1. Loh E, St John Sutton M, Wun CC, et al. Ventricular dysfunction and the risk of stroke after myocardial infarction. N Engl J Med. 1997;336:251-7.
2. Teo KK, Yusuf S, Pfeffer M, et al. Effects of long-term treatment with angiotensin-converting enzyme inhibitors in the presence or absence of aspirin: a systematic review. Lancet. 2002;360:1037-43.

3. Massie BM, Krol WF, Ammon SE, et al. The Warfarin and Antiplatelet Therapy in Heart Failure trial (WATCH): rationale, design, and baseline patient characteristics. J Card Fail. 2004;10:101-12.

4. Massie BM, Collins JF, Ammon SE, et al. Randomized trial of warfarin, aspirin and clopidogrel in patients with chronic heart failure: the Warfarin and Antiplatelet Therapy in Heart Failure (WATCH) Trial. Circulation. 2009;119:1616-24.

5. Cleland JGF, Findlay I, Jafri S, et al. The Warfarin/Aspirin Study in Heart failure (WASH): a randomized trial comparing antithrombotic strategies in patients with heart failure. Am Heart J. 2004;148:157-64.

6. Masoudi FA, Wolfe P. Havranek, et al. Aspirin use in older patients with heart failure and coronary artery disease: national prescription patterns and relationship with outcomes. J Am Coll Cardiol. 2005;46:955-62. 